American Journal of Biochemistry and Biotechnology 6 (2): 116-119, 2010

ISSN 1553-3468

(C) 2010 Science Publications

\title{
Total Level of Serum Homocysteine in Males and Females with Coronary Heart Disease of Different Age Groups
}

\author{
${ }^{1}$ Faisal I. Mohammad, ${ }^{2}$ Sally Awawdeh, ${ }^{3}$ Akram Saleh and ${ }^{4}$ Nabil A. Bashir \\ ${ }^{1}$ Department of Physiology and Biochemistry, Faculty of Medicine, \\ University of Jordan, Amman, Jordan \\ ${ }^{2}$ Medical Laboratory, Princess Badea' Hospital, Irbid, Jordan \\ ${ }^{3}$ Department of Internal Medicine, Faculty of Medicine, Cardiology Section, \\ University of Jordan, Amman, Jordan \\ ${ }^{4}$ Department of Biochemistry and Molecular Biology, Faculty of Medicine, \\ University of Science and Technology, P.O. Box 3030, Irbid 22110, Jordan
}

\begin{abstract}
Problem statement: Elevated plasma total homocysteine concentration is a risk factor of cardiovascular disease. Total homocysteine level is a strong predictor of mortality in-patient with an angiographically confirmed Coronary Heart Disease (CHD), so we want to know at what age in males and females elevated homocysteine will be significantly elevated and this will help for better management and prognosis by decreasing the level of homocysteine. Approach: Plasma level of homocysteine was determined in male and female patients below and above 50 years old, who have coronary heart disease with diabetes or without diabetes. Fifty two coronary heart diseases with type 2 diabetic patients and a matched number of healthy subjects as a control and another 52 coronary heart disease patients without diabetes were included in this study. Plasma homocysteine was determined by Enzymatic Immuno Sorbant Assay (ELISA). Results: Plasma homocysteine level in coronary heart disease diabetic male and female patients who are $<50$ years old was $10.3 \pm 2.5$ and $12.66 \pm 5.4 \mathrm{umol} \mathrm{L}^{-1}$, respectively, while it is $28.12 \pm 9.5$ and $29.44 \pm 4.3 \mathrm{umol} \mathrm{L}^{-1}$ in male and female patients $>50$ years old, respectively. Plasma homocysteine level in coronary heart disease nondiabetic male and female patients who are $<50$ years old was $12.72 \pm 8.5$ and $13.60 \pm 6$ umol $\mathrm{L}^{-1}$, respectively, while it is $26.9 \pm 11.1$ and $27.56 \pm 6.8$ umol $\mathrm{L}^{-1}$ in male and female patients $>50$ years old, respectively. Conclusion: It is concluded that plasma level of homocysteine is significantly elevated in diabetic coronary heart disease female patients above 50 years old and significantly elevated in nondiabetic coronary heart disease males and female patients, thus nondiabetic coronary heart disease male and female patients and diabetic coronary female patients are at high risk of vascular diseases. It is recommended that these patients may take supplementation of folate and vitamin B12 to reduce the level of homocysteine.
\end{abstract}

Key words: Coronary heart disease, homocysteine, diabetes, vitamin B, folate, age, male, female

\section{INTRODUCTION}

Homocysteine is not obtained from the diet (Selhub, 1999) Instead, it is biosynthesized from methionine via a multi-step process. Homocysteine can be recycled into methionine. This process uses N5methyl tetrahydrofolate as the methyl donor and cobalamin (Vitamin B12)-related enzymes. Deficiencies of the vitamins folic acid, pyridoxine (B6), or B12 (cyanocobalamin) can lead to high homocysteine levels (Miller et al., 1994).
Supplementation with pyridoxine, folic acid, or B12 reduces the concentration of homocysteine in the bloodstream (Van Guldener and Stehouwer 2001; Melinda, 2006). Increased levels of homocysteine are linked to high concentrations of endothelial asymmetric dimethylarginine. Recent research suggests that intense, long duration exercise raises plasma homocysteine levels, perhaps by increasing the load on methionine metabolism (Van Meurs et al., 2004).

Total homocysteine level is a strong predictor of mortality in-patient with an angiographically confirmed

Corresponding Author: Nabil A. Bashir, Department of Biochemistry and Molecular Biology, Faculty of Medicine, University of Science and Technology, P.O. Box 3030, Irbid 22110, Jordan 
coronary heart disease (Russo et al., 2004). Plasma total Homocysteine (tHcy) is commonly elevated in persons with diabetes. This may be due to effects of insulin and/or glucose and/or metabolic control on the metabolism or plasma levels of tHcy.

Hyperhomocysteinemia due either to inborn errors of metabolism or to nutritional deficiency of the vitamins involved in homocysteine metabolism is associated with suspected atherosclerosis and both arterial and venous thrombosis. Predominant among the causes of mortality and major morbidity is thromboembolism, followed by cerebrovascular accident, peripheral arterial thrombosis and myocardial infarction. Hyperhmocysteinemia, regardless of its cause, is an independent risk factor for stroke, coronary or peripheral artery disease (Russo et al., 2004). The objective of this study is to examine if there is an association between elevated plasma level of homocysteine with age and sex in diabetic and nondiabetic coronary heart disease patients.

\section{MATERIALS AND METHODS}

A total number of 104 subjects were included in this study. Fifty two patients showed electrocardiographic abnormalities suggestive of ischemia or previous asymptomatic myocardial infarction with Coronary Heart Disease (CHD) from both sexes who admitted at CCU in Princess Basma Teaching Hospital in Irbid City, the other 52 were age and sex matched controls that refereed to the lab of Princess Basma Teaching Hospital in Irbid City. Venous blood samples collected after $12 \mathrm{~h}$ of overnight fast into plain tubes and EDTA tubes, serum or plasma was obtained by low speed centrifuge at $1000 \mathrm{~g}$ for 15 min and samples were immediately separated into aliquot and stored at $-20^{\circ} \mathrm{C}$ until analysis. The subjects were interviewed and a questionnaire paper and consent were completed, which included sex, age, DM, hypertension and coronary heart disease CHD. Symptoms of coronary events as defined by Rose questionnaire, history of coronary events, stroke, claudication intermittens, heart failure, uncontrolled hypertension (>180/100 mmHg), cardiomyopathy, chronic or acute diseases, pregnancy, liver or kidney disease (creatinine $>130 \mu \mathrm{mol} \mathrm{L}^{-1}$ ), proteinuria (dipstick-positive proteinuria or Albumin Excretion Rate $\left(\right.$ AER) $\geq 300 \mathrm{mg} \mathrm{day}^{-1}$. Diabetes was diagnosed according to ADA criteria (American Diabetes Association, 1997). Hypertension was diagnosed according to WHO criteria (WHO-ISH, 1999). Homocysteine (Hcy) in blood was quantitatively estimated in plasma by Enzymatic Immunosorbant Assay (ELISA). Homocysteine Microplate Enzyme immunoassay provided by BIO-RAD (BIO-RAD, USA). Protein bound Hcyt was reduced by dithiotheritol to free Hcyt and enzymatically converted to S-Adenosyl-L-Homocysteine $(\mathrm{SAH})$ in a separate procedure prior to the immunoassay.

\section{RESULTS}

As shown in Table 1 plasma homocysteine level in coronary heart disease diabetic male and female patients who are $<50$ years old was $10.3 \pm 2.5$ and 12.66 \pm 5.4 umol $\mathrm{L}^{-1}$, respectively, while it is $28.12 \pm 9.5$ and $29.44 \pm 4.3$ umol $\mathrm{L}^{-1}$ in males and females patients $>50$ years old, respectively. As shown in Table 2 plasma homocysteine level in nondiabetic coronary heart disease male and female patients who are <50 years old was $12.72 \pm 8.5$ and $13.60 \pm 6$ umol $\mathrm{L}^{-1}$, respectively, while it is $26.9 \pm 11.1$ and $27.56 \pm 6.8$ umol $\mathrm{L}^{-1}$, respectively in patients $>50$ years old ( Table 2).

Table 1: Mean values and standard deviation of homocysteine plasma level in diabetic coronary heart disease CHD and control subjects

\begin{tabular}{|c|c|c|c|c|c|c|}
\hline \multirow[b]{2}{*}{ Parameter and age (years) } & \multicolumn{3}{|l|}{ Male } & \multicolumn{3}{|l|}{ Female } \\
\hline & Non CHD & $\mathrm{CHD}$ & $\mathrm{P}$ & Non CHD & CHD & $\mathrm{P}$ \\
\hline Hmcy $\left(\right.$ umol L ${ }^{-1}$ ) ages $>50$ & $19.47 \pm 10.8$ & $28.12 \pm 9.5$ & 0.11 & $15.56 \pm 4.8$ & $29.44 \pm 4.3$ & 0.00 \\
\hline Hmcy $\left(\right.$ umol L ${ }^{-1}$ ) ages $<50$ & $11.90 \pm 3.4$ & $10.3 \pm 2.5$ & 0.70 & $9.16 \pm 2.5$ & $12.66 \pm 5.4$ & 0.42 \\
\hline
\end{tabular}

CHD: Coronary Heart Disease; Hmcy: Homocysteine

Table 2: Mean values and standard deviation of homocysteine plasma level in CHD and control subjects

\begin{tabular}{|c|c|c|c|c|c|c|}
\hline \multirow[b]{2}{*}{ Parameter and age (years) } & \multicolumn{3}{|l|}{ Male } & \multicolumn{3}{|l|}{ Female } \\
\hline & Non CHD & CHD & $\mathrm{P}$ & Non CHD & CHD & $\mathrm{P}$ \\
\hline Hmcy $\left(\right.$ umol L ${ }^{-1}$ ) ages $>50$ & $18.41 \pm 8.6$ & $26.9 \pm 11.1$ & 0.02 & $18.28 \pm 6.2$ & $27.56 \pm 6.8$ & 0.00 \\
\hline Hmcy $\left(\right.$ umol L ${ }^{-1}$ ) ages $<50$ & $10.88 \pm 5.4$ & $12.72 \pm 8.5$ & 0.62 & $10.41 \pm 5.5$ & $13.60 \pm 6.6$ & 0.29 \\
\hline
\end{tabular}

CHD: Coronary Heart Disease; Hmcy: Homocysteine 


\section{DISCUSSION}

Elevations of homocysteine occur in the rare hereditary disease homocystinuria and in the methylene-tetrahydrofolate-reductase polymorphism genetic traits. The latter is quite common (about $10 \%$ of the world population) and it is linked to an increased incidence of thrombosis and cardiovascular disease, which occurs more often in people with above minimal levels of homocysteine (about $6 \mu \mathrm{mol} \mathrm{L} \mathrm{L}^{-1}$ ). These individuals require adequate dietary riboflavin in order for homocysteine levels to remain normal. Common levels in Western populations are 10-12 and levels of $20 \mu \mathrm{mol} \mathrm{L} \mathrm{L}^{-1}$ are found in populations with low Bvitamin intakes (e.g., New Delhi) or in the older elderly (e.g., Rotterdam, Framingham). Women have 10-15\% less homocysteine during their reproductive decades than men, which may help explain the fact they suffer myocardial infarction (heart attacks) on average 1015 years later than men.

Plasma level of homocysteine in Jordanian healthy control subjects below 50 years old is 6-15 umol L $\mathrm{L}^{-1}$ which is normal when compared to other populations, while it is $11-25 \mathrm{umol} \mathrm{L}^{-1}$ above 50 years old which is normal for old people.

Male and female diabetic or nondiabetic CHD patients below 50 years old have homocysteine level close to the control healthy subjects with no significant difference, this may indicate of no vitamin B or folate deficiencies and homocysteine does not represent a major risk factor for myocardial infarctions.

Plasma level in male and female nondiabetic CHD above 50 years old patients is significantly higher than the matched controls; this could be due to vitamin B and folate deficiencies or mutations in some genes that are responsible for specific enzymes which are important in homocysteine metabolism such as tetrahydrofolate-methyltransferase.

It was found that plasma level of homocysteine is significantly elevated in diabetic CHD female patients above 50 years old but not for females below 50 years old or males above 50 years old. Total homocysteine (tHcy) is commonly elevated in persons with diabetes (WHO-ISH, 1999). This may be due to effects of insulin and/or glucose and/or metabolic control on the metabolism of tHcy. Elevated level of homocysteine in females than males as shown here is in contradiction with what Nygard et al. (1995) and Fonseca et al. (1999) reported. Among males, age is not a significant predictor of homocysteine level, while in female's age, is a significant predictor of homocysteine. The elevated level of serum homocysteine in females $>50$ years old was found to be associated with high levels of serum lipids mainly LDL and triglycerides and low level of HDL (data is not shown), this may shed light on the role of estrogen on the metabolism of homocysteine at menopause and how this could form a risk factor of CHD in females at this age. In this study we are consistent with those of Russo et al. (2004) and colleagues (WHO-ISH, 1999), who found age and sex were significantly associated with homocysteine level in type 2 diabetes. There is clear evidence that homocysteine levels can be lowered by administration of supplemental folate, with or without pyridoxine. What is not yet established is whether lowering of homocysteine by increased intake of these vitamins results in a diminution of cardiovascular risk. Recently published data strongly supports a protective role for high folate and vitamin B6 intake against ischemic heart disease. The prospect of modifying risk of cardiovascular disease in the general population by simple nutritional intervention is appealing and could constitute a substantial advance in the control of a major cause of morbidity and mortality worldwide ameliorates the cardiovascular risk have yet to be determined.

\section{CONCLUSION}

It is concluded that plasma level of homocysteine is significantly elevated in diabetic coronary heart disease female patients above 50 years old and significantly elevated in nondiabetic coronary heart disease males and female patients, thus nondiabetic coronary heart disease male and female patients and diabetic coronary female patients are at high risk of vascular diseases. It is recommended that these patients may take supplementation of folate and vitamin B12 to reduce the level of homocysteine.

\section{ACKNOWLEDGMENT}

The researchers would like thank the deanship of research in Jordan University of science and technology for the financial support of this study.

\section{REFERENCES}

American Diabetes Association, 1997. Report of the Expert Committee on the Diagnosis and classification of diabetes mellitus. Diabetes Care, 20: 1183-1197. PMID: 9203460

Fonseca, V., S.C. Guba and L.M. Fink, 1999. Hyperhomocysteinemia and the endocrine system: Implications for atherosclerosis and thrombosis. Endocr. Rev., 20: 738-759. DOI: 10.1210/er.20.5.738 
Melinda, M., 2006. Homocysteine, B Vitamins and Heart Disease. Supplementschat. http://www.supplementschat.org/homocysteine-bvitamins-and-heart-disease.html

Miller, J.W., M.R. Nadeau, D. Smith and J. Selhub, 1994. Vitamin B-6 deficiency Vs folate deficiency: Comparison of responses to methionine loading in rats. Am. J. Clin. Nutr., 59: 1033-1039. PMID: 8172087

Nygard, O., S.E. Vollset, H. Refsum, I. Stensvold and A. Tverdal, 1995. Total plasma homocysteine and cardiovascular risk profile. hordaland homocysteine study. J. Am. Med. Assoc., 274: 1526-1533. DOI: 10.1001/jama.274.19.1526

Russo, G.T., A. Di Benedetto, C. Giorda, E. Alessi and G. Crisafulli, 2004. Correlates of total homocysteine plasma concentration in type 2 diabetes. Eur. J. Clin. Invest., 34: 197-204. DOI: 10.1111/j.1365-2362.2004.01319.x
Selhub, J., 1999. Homocysteine metabolism. Annu. Rev. Nutr., 19: 217-246. DOI: 10.1146/annurev.nutr.19.1.217

Van Guldener, C. and C.D. Stehouwer, 2001. Homocysteine-lowering treatment: An overview. Expert Opin. Pharmacother., 2: 1449-1460. PMID: 11585023

Van Meurs, J.B.J., R.A.M. Dhonukshe-Rutten, S.M.F. Pluijm, M. van der Klift and R. de Jonge et al., 2004. Homocysteine levels and the risk of osteoporotic fracture. N. Engl. J. Med., 350: 2033-2041. PMID: 15141041

WHO-ISH, 1999. Guidelines for the management of hypertension. J. Hypertens., 17: 151-183. 